\title{
Earthquake statistics in a Block Slider Model and a fully dynamic Fault Model
}

\author{
D. Weatherley and S. Abe \\ ACcESS, The University of Queensland, Brisbane, Australia
}

Received: 3 August 2004 - Revised: 29 October 2004 - Accepted: 11 November 2004 - Published: 17 November 2004

Part of Special Issue "Seismicity pattern dynamics"

\begin{abstract}
We examine the event statistics obtained from two differing simplified models for earthquake faults. The first model is a reproduction of the Block-Slider model of Carlson et al. (1991), a model often employed in seismicity studies. The second model is an elastodynamic fault model based upon the Lattice Solid Model (LSM) of Mora and Place (1994). We performed simulations in which the fault length was varied in each model and generated synthetic catalogs of event sizes and times. From these catalogs, we constructed interval event size distributions and inter-event time distributions. The larger, localised events in the BlockSlider model displayed the same scaling behaviour as events in the LSM however the distribution of inter-event times was markedly different. The analysis of both event size and interevent time statistics is an effective method for comparative studies of differing simplified models for earthquake faults.
\end{abstract}

\section{Introduction}

In order to understand the statistical behaviour of earthquakes, the knowledge of long earthquake sequences is necessary. However, the time for which instrumental records of earthquakes are available is relatively short compared to the recurrence time of major earthquakes. Thus artificial data generated from numerical simulation models are used.

The completely realistic simulation of the full earthquake cycle for a time sufficient to generate enough data is currently computationally infeasible. Therefore, a wide variety of models with a varying degree of simplification have been developed. Those models range from fully dynamic fault models which can realistically model a few slip events on a single fault to cellular automaton (CA) models which are able to simulate long sequences of events for a fault or a fault system in a highly simplified way.

Correspondence to: D. Weatherley

(dion@quakes.uq.edu.au)
In order to investigate how well highly simplified models capture the behaviour of the more realistic models we have compared the event statistics of a Block-Slider model and a fully dynamic model of a single fault based on the Lattice Solid Model (LSM). Block-Slider model was selected for comparison with the LSM, as opposed to a more simplified CA model, so that comparisons of event statistics would be easier. The measure for event size most commonly used for CA models is rupture area rather than energy release. Computation of rupture areas is difficult in dynamic rupture models and computation of energy release is often not possible in CA models (depending upon the design of the models). The inertial Block-Slider model is sufficiently similar to the LSM that a common measure of event size may be employed for comparisons of event statistics.

The paper is organised as follows. Sections 2 and 3 describe the LSM and Block-Slider models and the numerical experiments performed with each model. In Sect. 4 we describe a method for detecting dynamic ruptures and computing the size of events. This method is used to generate event catalogs with which to compute frequency-size distributions and inter-event time distributions. The results from the two models are compared and major conclusions are outlined in Sect. 5.

\section{The dynamic fault model}

\subsection{The method}

The Lattice Solid Model (Mora and Place, 1994; Place and Mora, 1999) is a particle based model similar to the Discrete Element Model (DEM) developed by Cundall and Strack (1979). The model consists of spherical particles which are characterized by their radius $r$, mass $m$, position $\mathbf{x}$ and velocity $\mathbf{v}$. The particles interact with their nearest neighbors, e.g. by elastic and frictional forces. The particles can be linked together by elastic bonds or springs (Fig. 1), in which case the elastic forces are attractive or repulsive, depending 


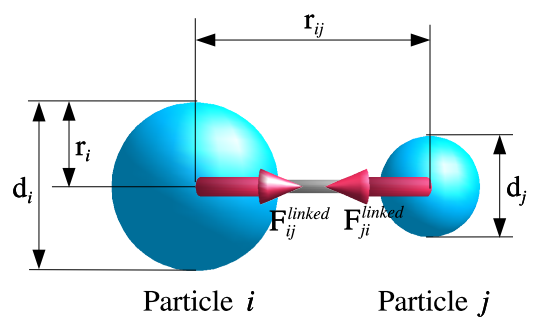

Fig. 1. Attractive forces between linked particles. $\mathbf{F}_{i j}$ is the force applied to particle $i$ due to the interaction with particle $j$ whereas $\mathbf{F}_{j i}$ is the force applied to particle $j$ due to the interaction with particle $i$.

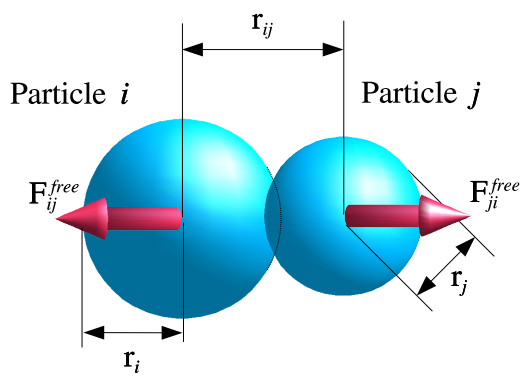

Fig. 2. Repulsive forces between particles which are not linked together.

on whether the particles are closer or more distant than the equilibrium distance $\left(r_{0}\right)_{i j}$.

$$
\mathbf{F}_{i j}^{\text {linked }}= \begin{cases}k_{i j}\left(r_{i j}-\left(r_{0}\right)_{i j}\right) \mathbf{e}_{i j} & r_{i j} \leq\left(r_{c u t}\right)_{i j} \\ \mathbf{0} & r_{i j}>\left(r_{c u t}\right)_{i j},\end{cases}
$$

where $k_{i j}$ is the spring constant for the elastic interaction between the particles, $r_{i j}$ is the distance between the particles $i$ and $j,\left(r_{c u t}\right)_{i j}$ the breaking distance for the link between the particles and $\mathbf{e}$ is a unit vector in the direction of the interaction.

Links are broken if the distance between the particles exceeds the threshold breaking distance $\left(r_{c u t}\right)_{i j}$. If two particles are not linked together (Fig. 2) the elastic force $\mathbf{F}_{i j}^{\text {free }}$ between the particles $i$ and $j$ is purely repulsive

$$
\mathbf{F}_{i j}^{\text {free }}= \begin{cases}k_{i j}\left(r_{i j}-\left(r_{0}\right)_{i j}\right) \mathbf{e}_{i j} & r_{i j} \leq\left(r_{0}\right)_{i j} \\ \mathbf{0} & r_{i j}>\left(r_{0}\right)_{i j} .\end{cases}
$$

Two unbonded interacting particles can be in static or dynamic frictional contact (Fig. 3). The force on particle $i$ due to the dynamic frictional contact with particle $j$ is given by

$$
\mathbf{F}_{i j}^{D}=-\mu F_{i j}^{n} \mathbf{e}_{\mathbf{i j}}^{T},
$$

where $\mu$ is the coefficient of friction between the particles, $F_{i j}^{n}$ is the magnitude of the normal force and $\mathbf{e}_{\mathbf{i j}}{ }^{T}$ is a unit vector in the direction of the relative tangential velocity between the particles (Cundall and Strack, 1979; Mora and Place, 1998; Place and Mora, 1999).

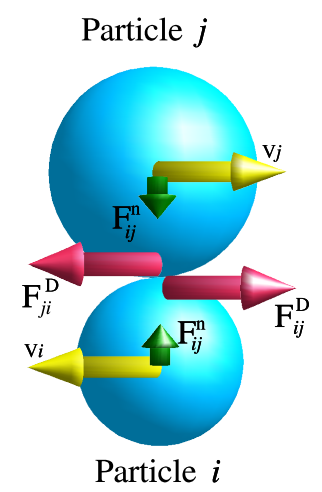

Fig. 3. Frictional forces between particles which are not linked together.

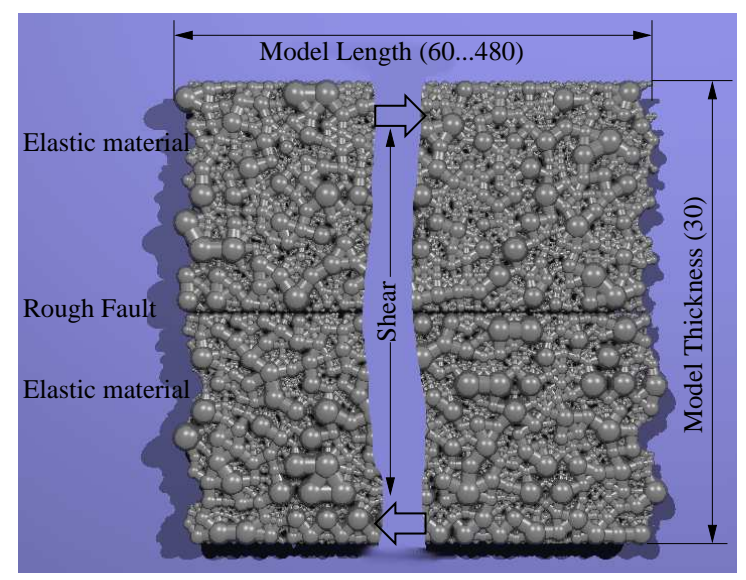

Fig. 4. Particle Model of a 1-D Fault embedded in a 2-D medium.

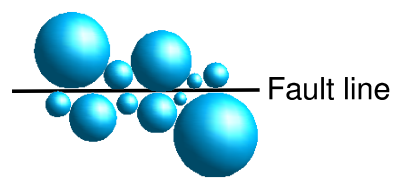

Fig. 5. Detail of a rough planar 1-D Fault.

\subsection{The model setup}

The model used for the particle based simulation of fault slip consists of two 2-D blocks of elastic material, formed by a dense packing of particles which are fully linked together and a rough fault in between (Fig. 4). In order to enable large total displacements of the fault in models of a limited size periodic boundary conditions have been applied in the direction of the shear movement.

The rough fault is generated by fitting random sized circular particles to a line (Fig. 5).

The assembly is then compressed in the direction perpendicular to the fault to maintain a normal force and sheared in the direction parallel to the fault at a constant velocity. The boundary conditions in shear direction are circular, thus 
arbitrary amounts of shear displacement are possible. The roughness along the fault surface is sufficient to cause to model to move in a stick-slip motion when sheared even without any changes in the coefficient of friction between static and dynamic friction.

\subsection{The numerical experiments}

A number of numerical experiments with different model setups have been performed. In all models the radii of the particles $r_{i}$ range from 0.1 to 1.0 model units. The material parameters of the model are held constant for all the simulations:

$$
\begin{aligned}
& \mathrm{m}_{0}=1.0 \\
& \mathrm{k}_{i j}=1.0 \\
& \mu=0.6,
\end{aligned}
$$

where $m_{0}$ is the mass of a particle of radius $1.0, k_{i j}$ is the spring constant for the particle interaction (Eqs. 1,2) and $\mu$ is the intrinsic friction between particles along the fault (Eq. 3). The breaking distance $\left(r_{c u t}\right)_{i j}$ has been chosen sufficiently large so that no breaking of bonds occurs during the simulations.

The geometry of the model was varied between the different simulations. While the width of the model perpendicular to the fault was held constant at 30 model units, the length of the model in direction of the fault was varied between 120 and 1890 model units. Models of length 120, 240, 480, 945, 1440 and 1890 model units have been used, resulting in models consisting of between $\approx 7800$ and $\approx 122000$ particles. The driving velocity applied to the edges of the model was 0.00025 model velocity units on each driving plate, or a total shear velocity of 0.0005 model units for all experiments.

For a regular 2-D lattice the P-wave velocity in the model can be calculated as

$$
v_{p}=\sqrt{\frac{9}{8}} r_{0} \sqrt{\frac{k}{m_{0}}} .
$$

It has been shown by Place and Mora (2001) that this relation also applies to the random lattice used here. Thus the P-Wave velocity in the models used is $v_{p} \approx 2.1$ and therefore the applied shear velocity is equivalent to approximately $0.025 \% v_{p}$. This value is several orders of magnitude larger than realistic tectonic loading speeds for earthquake faults, but in order to generate a sufficient number of events a compromise had to be found between more realistic driving speeds and limited simulation time.

The simulations were performed for $1-3 \times 10^{7}$ time steps, resulting in the generation of between 3000 and 8000 events generated during each simulation.

In order to minimize the computing time necessary for the simulations, a parallel implementation of the Lattice Solid Model (Abe et al., 2004) was used, utilising up to 42 CPUs of an SGI Altix 3700 for the largest models.

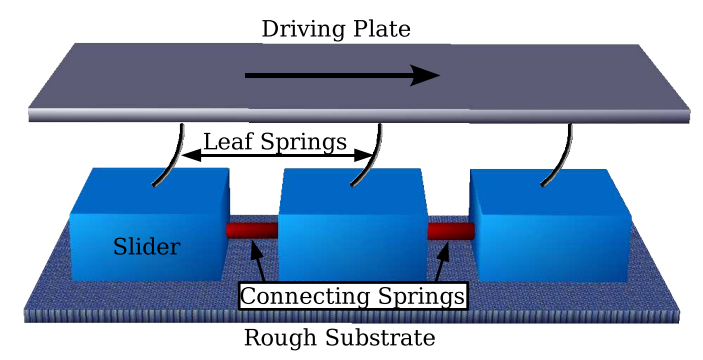

Fig. 6. 1-D block-slider model.

\section{The block-slider model}

\subsection{The model}

The block-slider model is a reproduction of that of Carlson et al. (1991). The model consists of a 1-D chain of $N$ blocks connected via linear elastic springs with spring constant $K_{c}$ (Fig. 6). Each block is also connected to a rigid driving plate via leaf springs with constant $K_{l}$. The driving plate is moved at a constant speed $V_{p l}$ and blocks interact with a rough substrate via a velocity-weakening friction law (described below in Eq. 6).

The partial differential equation to be solved is the following:

$$
\begin{gathered}
\ddot{U}_{i}(t+\Delta t)=\frac{K_{c}}{M}\left[U_{i+1}(t)-2 U_{i}(t)+U_{i-1}(t)\right] \\
+\frac{K_{l}}{M}\left[V_{p l} t-U_{i}(t)\right]+\phi(i, t),
\end{gathered}
$$

where $i=0,1, \ldots, N-1$ is the block index, $M$ is the mass of each block ( $M=1$ in the following), and $\phi(i, t)$ is the friction law. We have refrained from using the non-dimensionalised form of the PDE to facilitate comparison of parameter values with the equivalent LSM parameters.

The velocity-weakening friction law governing interactions between individual blocks and the rough substrate is given by:

$\phi(i, t)=\left\{\begin{array}{ll}\frac{\mu_{0 i}}{1+\dot{U}_{i}(t)} & \text { if } \dot{U}_{i}(t) \geq 0 \\ {\left[-\infty, \mu_{0 i}\right]} & \text { otherwise }\end{array}\right.$,

where $\mu_{0 i}$ is the static coefficient of friction of the i-th block. Friction coefficients are selected from a uniform, random distribution with values in the range $[0.5,1.0]$. A friction law of this form prevents back-slip of sliding blocks, a situation that more closely reproduces frictional sliding in the LSM than do laws permitting back-slip such as Carlson and Langer (1989).

The time-integration scheme is an explicit finite-difference scheme that assumes constant acceleration during a given timestep $(\Delta t)$, hence the equations for updating displacements and velocities are as follows:

$U_{i}(t+\Delta t)=U_{i}(t)+\Delta t \dot{U}_{i}(t)+\frac{\Delta t^{2}}{2} \ddot{U}_{i}(t+\Delta t)$
$\dot{U}_{i}(t+\Delta t)=\dot{U}_{i}(t)+\Delta t \ddot{U}_{i}(t+\Delta t)$ 


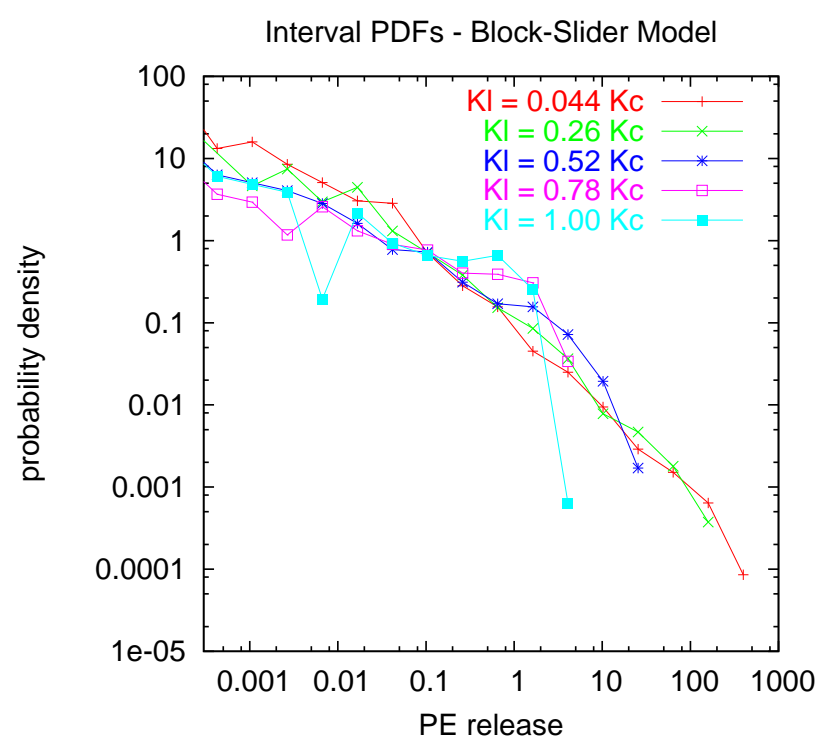

Fig. 7. Event Distributions for different values of the stiffness $K_{l}$ in the block-slider model.

We employ a circular boundary condition (so that block $N$ is equivalent to block 0 ) and all blocks are initially in their equilibrium positions $\left(\forall i, U_{i}=0\right)$.

Numerical experiments in which the fault length was varied, were also performed using the Block-Slider model. Models containing 256, 614, 972, 1450 and 1928 blocks were examined. Following the direction of Carlson et al. (1991), the leaf spring constant was set to $K_{l}=1 \% K_{c}$ and $K_{c}=1.0$. The choice of $K_{l}=1 \% K_{c}$ resulted in power-law scaling of events whereas for significantly larger values of $K_{l}$ the event size distribution would show a different behaviour (Fig. 7).

A plate velocity of approximately $1 \%$ the compressional wave-speed was selected. While this is higher than that of the LSM simulations, it is sufficiently small that stable sliding is inhibited. Simulations consisting of 10 million timesteps were performed, resulting in between 3000 and 5000 events.

\section{Results}

The aim of the present investigation is to determine whether the event statistics of LSM simulations are comparable to those obtained from Block-Slider simulations. We have focused upon the interval frequency-size distributions and inter-event time distributions. In order to compare the event statistics of the two models, it was necessary to devise a method for detection of events and computation of event sizes that could be applied to simulation data from either model. The method is described in the following subsection, subsequent to which the frequency-size and inter-event time distributions are presented.

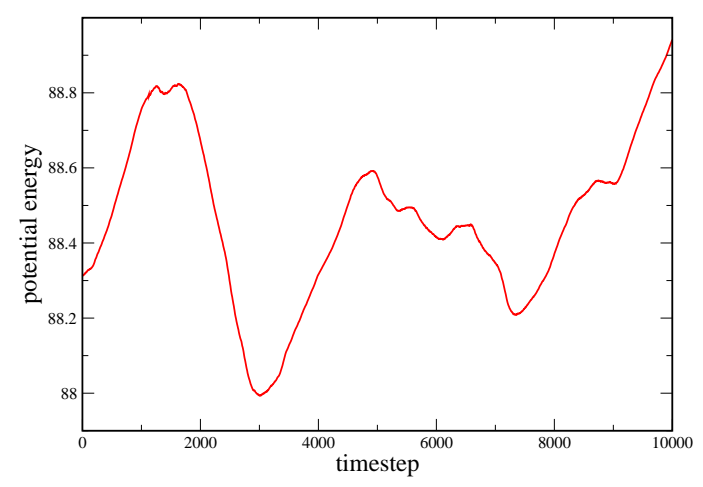

Fig. 8. Example of a potential energy time series obtained from the LSM.

\subsection{Computation of event sizes and PDFs}

There are a number of measures for the size of earthquakes. The measure most appropriate for use in simulations is the seismic moment (the rupture area multiplied by the mean slip). Computation of the seismic moment of events is somewhat complicated for inertial models. In inertial models it is often difficult to identify when dynamic rupture commences and ceases due to continuous aseismic creep of portions of the model fault or precursory aseismic creep prior to dynamic rupture. This complicates computation of mean slip and rupture area in a given event and hence, the seismic moment.

We investigated the possibility of selecting a lower velocity cut-off, above which a fault segment is presumed to be slipping, however could find no criterion for selecting this cut-off that was not purely arbitrary. The distribution of slip velocities was monotonic, decreasing for all slip velocities. The magnitude of the cut-off velocity might also need to be varied for simulations with differing driving rates or viscosities. This would further complicate estimation of event sizes in simulations with differing model parameters.

We have elected to use a method for estimating event size based upon detection of drops in the potential energy timeseries. The potential energy is computed as the sum of the potential energies of all elastic springs in the particle model and as the sum of the potential energies of all elastic and leaf springs in the block-slider model. During intervals in which the fault is locked, the potential in the model increases due to loading at the boundaries. A slip event causes some of the potential energy to be converted into kinetic energy, resulting in a drop in the total potential energy of the model.

The change in potential energy should be proportional to the seismic moment. The method for detecting events and computing event sizes is as follows:

1. At each timestep the total elastic potential energy is recorded (Fig. 8).

2. The PE timeseries is postprocessed to compute the change in PE each timestep (blue curve in Fig. 9). 


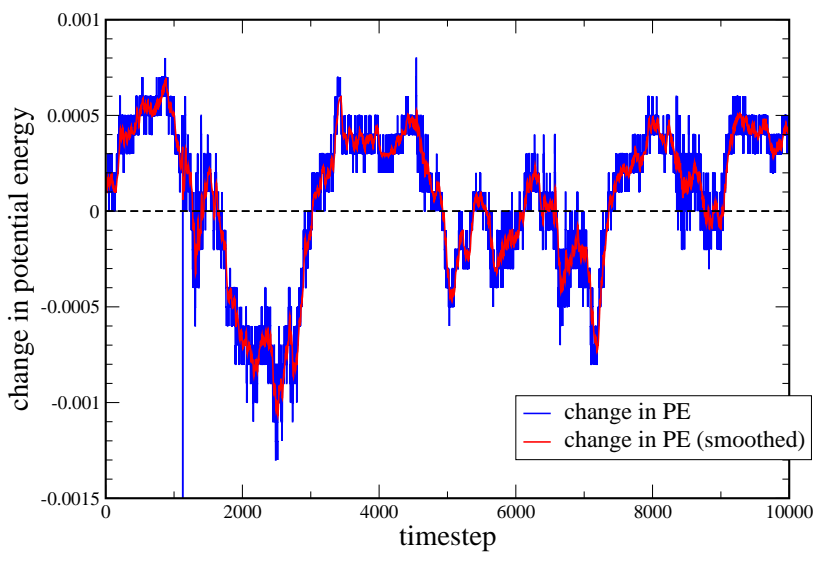

Fig. 9. Change in potential energy computed from the time series shown in Fig. 8.

3. The average change in PE is computed using leaky integration to smooth the change in PE time-series (red curve in Fig. 9).

$$
\Delta P E_{\text {avg }}(t+1)=\frac{P E(t+1)-P E(t)+\lambda \Delta P E_{\text {avg }}(t)}{1+\lambda t}
$$

4. The commencement of an event occurs when the average change in PE goes negative (beginning of gray area in Fig. 10) and the cessation of the event occurs when the average change in PE goes positive (end of gray area in Fig. 10).

5. The event size is computed as the net change in the PE from the commencement to cessation of the event (Fig. 11).

The leakiness parameter $(\lambda)$ controls the smoothing of the change in PE time-series. The value of this parameter $(\lambda=0.99)$ was selected by comparing the original PE timeseries with a plot of the detected times for commencement and cessation of events. A smaller value of $\lambda$ resulted in detection of spurious small events and a value of $\lambda$ closer to unity resulted in poor detection of the cessation times of events and hence, poor estimation of the total change in potential energy.

As demonstrated later this approach provides an effective method for computing event sizes in both models. However, the total elastic potential energy is computed differently in each model. Therefore the absolute sizes of events can not be directly compared between the models. Despite this drawback we consider this method superior to comparisons based on different measures of event size such as potential energy release and number of slipping blocks.

In the following sections we present distributions of event sizes and inter-event times. Because of the multiple scales involved, the distributions must be carefully constructed. We employ a log-binning method (Corral (2004)) in which the bin sizes are defined so that the probability density is exponentially growing as $c^{n}$ where $c>1$ and $n$ labels consecutive

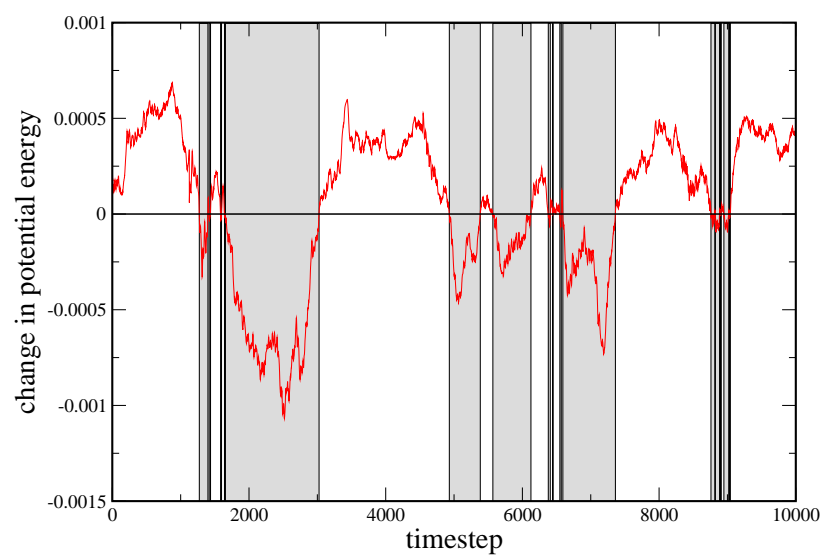

Fig. 10. Smoothed change of potential energy with picked events shown in gray.

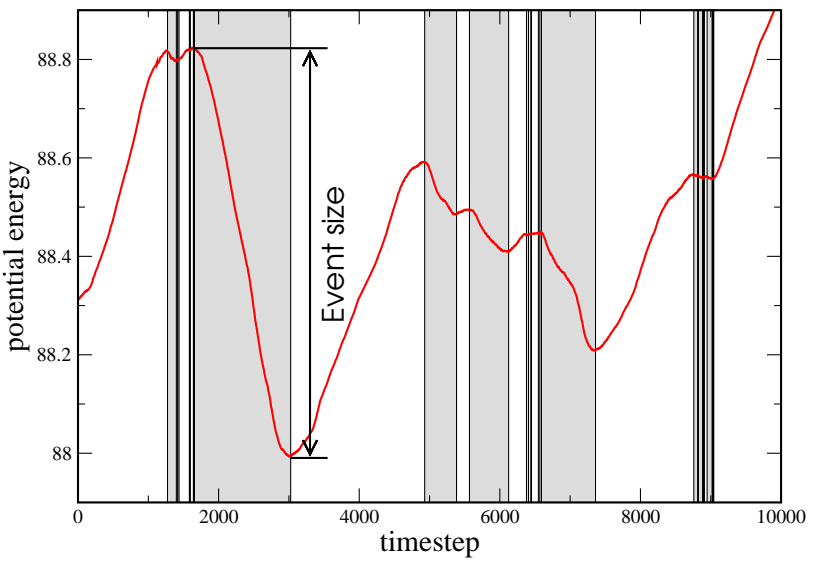

Fig. 11. Original potential energy time series with picked events (in gray) and calculation of event size illustrated.

bins. The method ensures an appropriate bin size for each scale. The number of samples in each bin is normalised by the bin-size and the total number of samples, to obtain an estimate of the probability density over each bin.

\subsection{Event size distributions}

An event size catalog was constructed for each simulation, using the event picking method above. The event catalogs were then used to construct PDFs of event sizes. Results from LSM simulations are given in Fig. 12 and from blockslider simulations in Fig. 13. Events smaller that $10^{-4}$ have been omitted from these figures due to statistical scatter in the data.

The PDFs obtained from LSM simulations may be fitted by a power-law relation with exponent 1.07 for approximately 3 decades. The event size PDFs from block-slider simulations also display a power-law segment spanning approximately 3 decades with an exponent of approximately 1.0. However the PDFs deviate from this power-law relation for both small and large event sizes. Small events roughly 


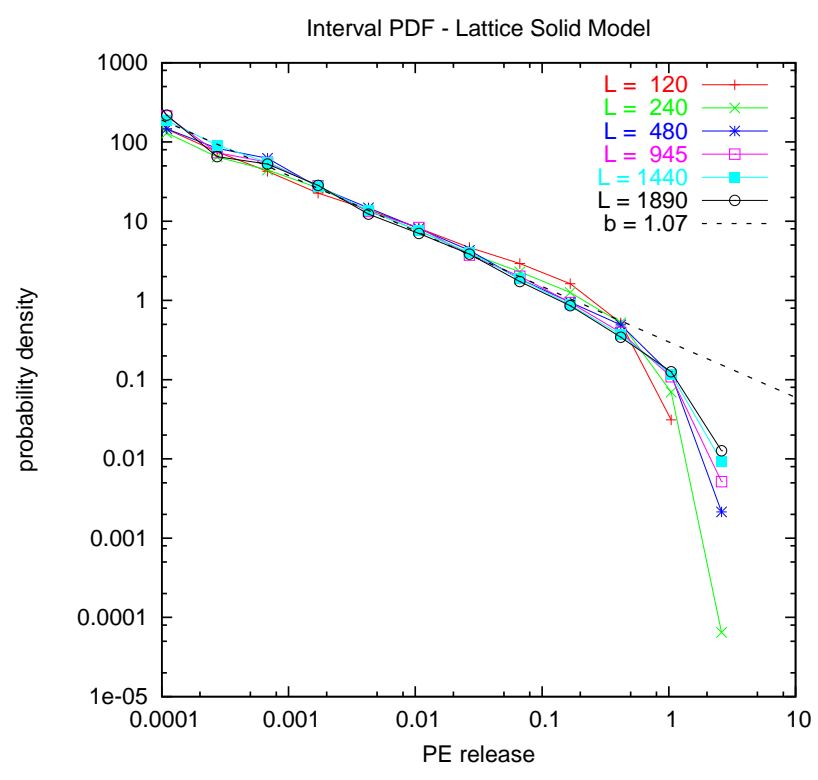

Fig. 12. Event size PDFs for LSM models with differing fault lengths.

follow a power-law relation with an exponent of approximately 0.5 although there is considerable scatter.

The differing power-law relations for small and moderatesized events in the block-slider model may be a manifestation of the differing dynamical nature of the events. Carlson and Langer (1989) identified three classes of events in the block-slider models termed "microscopic", "localized" and "delocalized". Microscopic events involve only one or a few blocks whereas localized events involve propagating rupture fronts that cause a number of blocks to slip during the event. A localized event does not however, cause slip of all of the blocks (a delocalized event).

Due to the random distribution of friction coefficients, we do not obtain "delocalized" events, however microscopic and localized events are expected. We hypothesise that the differing dynamical nature of small and moderate-sized events results in differing power-law exponents (and hence differing scaling behaviour) in the event size PDFs.

Interestingly, the scaling of localized events in the blockslider simulations is almost identical to the scaling observed in the LSM simulations. We suspect that the non-existence of microscopic events in the LSM is due to the stronger elastic coupling afforded by the bulk material surrounding the fault. When slip of a single frictional contact occurs, the strong elastic coupling promotes propagation of rupture along the fault.

Both LSM and block-slider simulations display an exponential roll-off in event numbers for the large event tail of the distributions. For both models the amount of roll-off decreases as the model size increases, suggesting that the rolloff is a finite size effect. To be certain that the roll-off is a finite size effect, it would be necessary to determine if the event size distribution continues to scale to large sizes as the

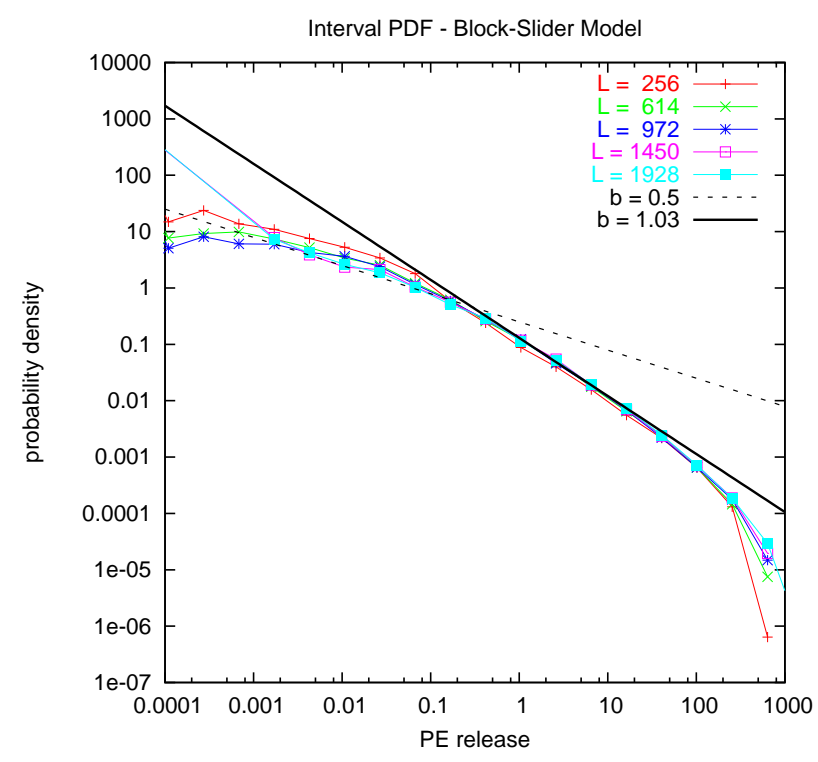

Fig. 13. Event size PDFs for block-slider models with differing fault lengths.

fault length increases. Given the computational burden of performing simulations with longer faults, this experiment has been delayed to a future investigation.

\subsection{Inter-event time distributions}

We also examined the distribution of inter-event times for each simulation. Corral (2004) demonstrated that the probability densities of earthquake inter-event times follow a universal distribution, for different magnitude ranges and spatial regions. The universal distribution is a generalised gamma distribution with the following form:

$$
f(\theta)=C \frac{1}{\theta^{1-\gamma}} \exp \left(-\theta^{\delta} / B\right) .
$$

Corral (2004) performed a fit of this distribution to seismicity data and obtained the values for the parameters listed in Table 1 . The distribution function with these parameter values was found to be universal for seismicity. The parameter values were independent of the magnitude range, spatial region and tectonic environment.

The apparent universality of this distribution function prompted our analysis of the inter-event time distributions obtained from the LSM (Fig. 14) and Block-Slider (Fig. 15) simulations. The parameter values obtained from a fit of Eq. 9 are listed in Table 1.

The distribution function provides a good fit for simulation data from both models however the parameter values are not in agreement. While different simulations from a given model all display the same inter-event time distribution, different models have different distributions. This would suggest that the two models reside in differing universality classes. This result is not unexpected given that Block-Slider 


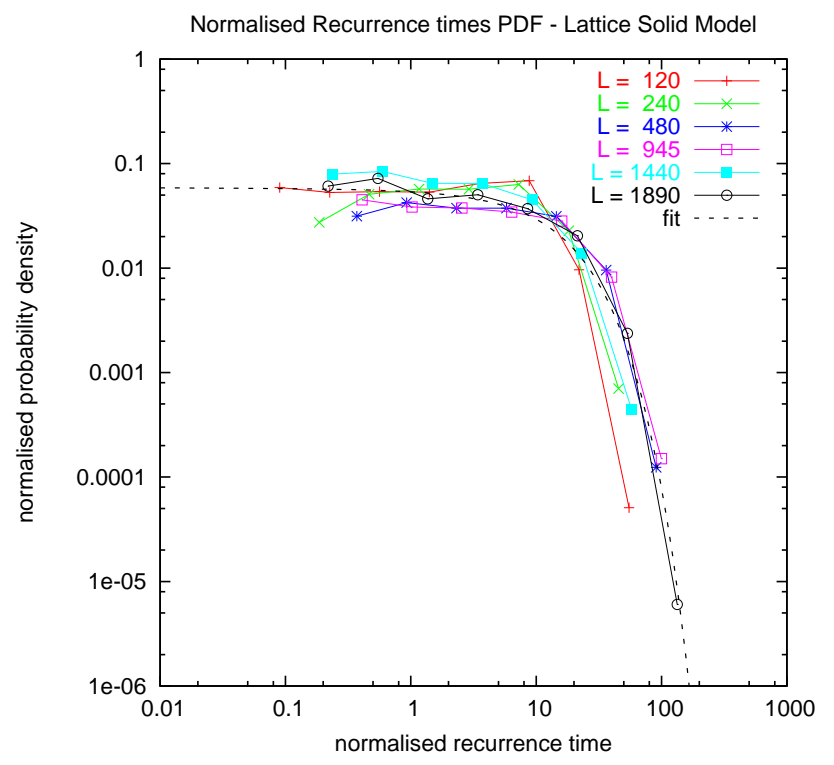

Fig. 14. Probability densities of inter-event times for LSM simulations.

Table 1. table of parameter values obtained from a fit of the generalised gamma distribution (Eq. 9) to inter-event time PDFs from seismicity (Corral, 2004), LSM simulations and Block-Slider simulations.

\begin{tabular}{cccc}
\hline parameter & seismicity & LSM & Block-Slider \\
\hline$\gamma$ & 0.67 & 0.995 & 0.0 \\
$\delta$ & 0.98 & 1.00 & 1.00 \\
$B$ & 1.58 & 15.21 & 100.0 \\
$C$ & 0.50 & 0.0573 & 0.25 \\
\hline
\end{tabular}

simulations contain additional microscopic events that do not occur in LSM simulations. Given that the results of Corral (2004) were obtained from distributed seismicity rather than earthquakes occurring within a single fault zone, the nonagreement between seismicity and the simulated data might also be expected.

Assuming that the inter-event time distribution for earthquakes is universal as attested by Corral (2004), analysis of the inter-event time distributions of differing earthquake models may provide a criterion for selection of models that best reproduce natural seismicity. For a simulation model to be useful as a tool for estimating the seismic hazard in a given region, it is critical that the model reproduces the inter-event time distribution of seismicity.

\section{Conclusions}

We have investigated the event statistics obtained from simulations of two differing models for earthquake faults. BlockSlider models with differing numbers of slider blocks display

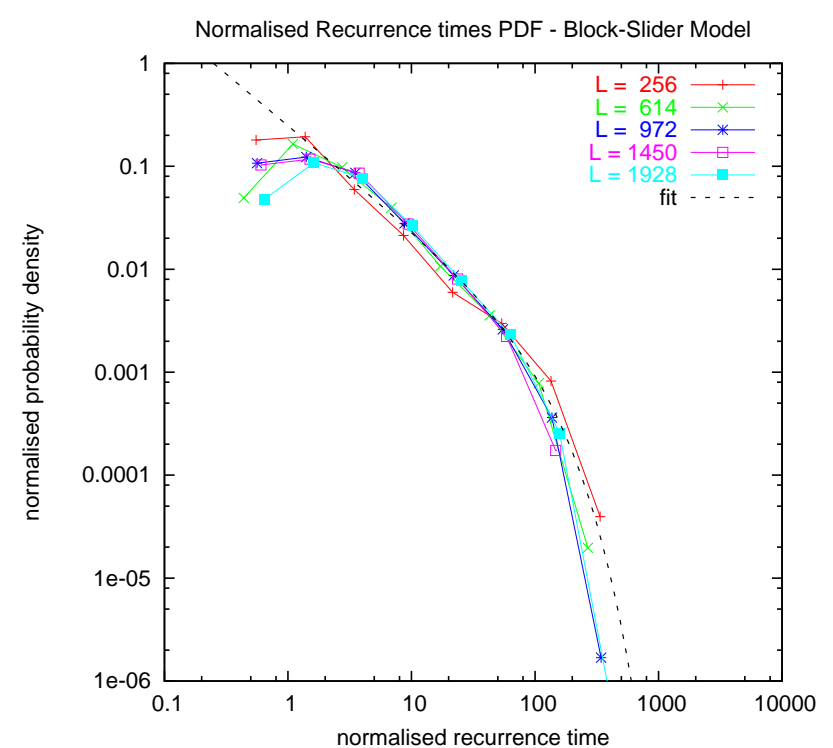

Fig. 15. Probability densities of inter-event times for Block-Slider simulations.

two different classes of events with differing scaling behaviour. These classes of events are microscopic events involving only one or a few blocks and localised dynamic rupture events.

The scaling of localised events in Block-Slider simulations is identical to the scaling of events in LSM simulations. The LSM simulations do not produce microscopic events. This is most likely due to the stronger elastic coupling in the bulk material. Slip at a single contact will in most cases, trigger dynamic rupture of neighbouring contacts.

We also examined the inter-event time distributions of both models and compared the results with that of natural seismicity. While all simulations for a given model display the same inter-event time distributions, suggesting universal behaviour, simulations from different models display markedly different distributions. We conclude that the two models reside in different universality classes to each other and to natural seismicity.

These results highlight the care that must be taken when attempting to understand long-term seismicity by analysis of more simplified models. Assuming that the inter-event time distribution of seismicity is universal (as suggested by Corral, 2004), analysis of such distributions for more simplified models may provide a method for categorising different earthquake models and evaluating their effectiveness.

Acknowledgements. Support is gratefully acknowledged by the Australian Computational Earth Systems Simulator Major National Research Facility, the Queensland State government, The University of Queensland, and SGI. The ACcESS MNRF is funded by the Australian Commonwealth Government and participating institutions (Univ. of Queensland, Monash U., Melbourne U., VPAC, RMIT, ANU) and the Victorian State Government. Computations were made using the ACcESS MNRF supercomputer, a 208 processor 1.1 TFlops SGI Altix 3700 which was funded by the 
Queensland State Government Smart State Research Facility Fund and SGI.

Edited by: Ch. Goltz

Reviewed by: S. Hainzl and S. Hergarten

\section{References}

Abe, S., Place, D., and Mora, P.: A Parallel Implementation of the Lattice Solid Model for the Simulation of Rock Mechanics and Earthquakes, Pure. Appl. Geophys., 161, in print, 2004.

Carlson, J. and Langer, J.: Mechanical Model of an Earthquake Fault, Phys. Rev. A, 40, 6470-6484, 1989.

Carlson, J., Langer, J., Shaw, B., and Tang, C.: Intrinsic Properties of a Burridge-Knopoff Model of an Earthquake Fault, Phys. Rev. A, 44, 884-897, 1991.
Corral, A.: Long-Term Clustering, Scaling, and Universality in the Temporal Occurrence of Earthquakes, Phys. Rev. Lett., 92, 108501, 2004.

Cundall, P. A. and Strack, O. D. A.: A Discrete Numerical Model for Granular Assemblies, Geótechnique, 29, 47-65, 1979.

Mora, P. and Place, D.: Simulation of the Stick-Slip Instability, Pure Appl. Geophys., 143, 61-87, 1994.

Mora, P. and Place, D.: Numerical Simulation of Earthquake Faults with Gauge: Towards a Comprehensive Explanation for the low Heat Flow, J. Geophys. Res., 103, 21 067-21 089, 1998.

Place, D. and Mora, P.: The Lattice Solid Model to Simulate the Physics of Rocks and Earthquakes: Incorporation of Friction, J. Comp. Physics, 150, 332-372, 1999.

Place, D. and Mora, P.: A Random Lattice Solid Model for Dimulation of Fault Zone dynamics and Fracture Processes, in: Bifurcation and Localization, Theory for Soils and Rocks, edited by: Mühlhaus, H. B., Dyskin, A. V., and Pasternak, E., Balkema, Rotterdam/Brookfield, 2001. 